\title{
ASOCIACIÓN DE LA FO, DURACIÓN E INTENSIDAD EN EL HABLA DE UNA MUJER DE MEDELLÍN (COLOMBIA) EN FUNCIÓN DE LA MODALIDAD ORACIONAL Y SUS SINTAGMAS
}

\author{
FO, DURATION AND INTENSITY ASSOCIATION IN COLOMBIAN \\ SPANISH FEMALE OF MEDELLIN ON FUNCTION OF SENTENCE \\ MODALITY AND ITS SYNTAGMAS
}

\author{
MERCEDES AMPARO MUNETTÓN \\ Universidad de Antioquia, Medellín \\ mercedes.muneton@udea.edu.co
}

\section{RESUMEN}

El presente trabajo se ha realizado en el marco del proyecto AMPER-Col (Atlas Multimedia de Prosodia del Espacio Románico en Colombia). El objetivo es analizar la asociación de los parámetros acústicos de frecuencia fundamental, de duración e intensidad en oraciones declarativas e interrogativas de una mujer de la zona urbana de Medellín. Para ello, se consideraron las diferencias mínimas perceptivas en cada uno de los parámetros y se usaron tablas de contingencias debido a que permiten ver en primer lugar la fuerza de asociación entre los parámetros y, en segundo lugar, si la asociación está por encima del azar. Los resultados principales muestran que los parámetros coocurren por encima del azar, mostrando un patrón de asociación entre ellos. Además, estos patrones diferencian las oraciones declarativas de las interrogativas.

Palabras clave: Prosodia, frecuencia fundamental, duración, intensidad, asociación.

\section{ABSTRACT}

This research has been carried out within the framework of AMPER-Col (Multimedia Atlas of Prosody of the Romanic Space in Colombia). The objective is to analyze the

* El trabajo se vincula a dos proyectos de investigación: Relaciones entre el límite prosódico y el límite sintagmático en oraciones declarativas e interrogativas de voces femeninas y masculinas de Colombia e Islas Canarias (España), Universidad de Antioquia, Comité para el Desarrollo de la Investigación (CODI), IP Mercedes Muñetón Ayala y Estudio comparativo de la entonación y del acento en zonas fronterizas del español (FFI2014-52716-P), proyecto de I+D del Programa Estatal de Fomento de la Investigación Científica y Técnica de Excelencia, Subprograma Estatal de Generación del Conocimiento del Ministerio de Economía y Competitividad de España, IP Josefa Dorta. La autora del trabajo es investigadora de ambos proyectos. 
association between the different acoustic parameters such as F0, duration and intensity in the prosody of declarative and interrogative sentences of a woman belonging to the urban zone of Medellín. For that, it was taken into account the just noticeable differences and it was used contingency tables in order to observe, firstly, the association between then and, secondly, if the association co-occur above chance level. Results showed that above chance the prosody parameters co-occur and those are different for both modalities.

Keywords: Prosody, fundamental frequency, duration, intensity, co-occurrences.

Recibido: 29.06.2016. Aceptado: 02.05.2017.

\section{INTRODUCCIÓN}

Ll presente trabajo se ha realizado en el marco del proyecto internacional AMCPER (Atlas Multimedia de Prosodia del Espacio Románico ${ }^{1}$ ), específicamente AMPER-Col (AMPER-Colombia ${ }^{2}$ ). El macro-proyecto surge en los albores del siglo XXI ante la ausencia de estudios orientados a la descripción y comparación de los patrones entonativos de las diferentes lenguas habladas en el espacio románico (Romano y Contini, 2001). El objetivo general del proyecto AMPER es el análisis prosódico de las lenguas románicas en frases enunciativas e interrogativas en voces femeninas y masculinas de zonas urbanas y rurales, utilizando un enfoque teórico y metodológico uniforme (Martínez Celdrán y Fernández Planas, 2006). Paralelamente, el objetivo de AMPER-Col es llenar un vacío en el análisis prosódico del habla colombiana debido a que actualmente se cuenta con pocos trabajos de investigación en esta área (Muñetón y Dorta, 2015).

Si bien es cierto la prosodia comprende diferentes elementos del habla como la entonación, la acentuación y los patrones rítmicos que adquieren unas características específicas debido a variaciones en parámetros acústicos de frecuencia fundamental (F0), intensidad y duración, tradicionalmente la mayoría de los trabajos se ha centrado en el análisis de la frecuencia fundamental (Dorta, Hernández y Díaz, 2009; Escandell, 2011). Esta tendencia está cambiando en los últimos años, puesto que uno de los objetivos del proyecto AMPER es el análisis de los tres parámetros acústicos mencionados. Precisamente, en este marco se ubica el presente estudio en el que se exploran los aspectos entonativos, temporales y de intensidad en oraciones declarativas e interrogativas en el habla de una mujer urbana de Me-

${ }^{1}$ Los coordinadores generales hasta mediados de 2015 han sido Michel Contini (Université Stendhal-Grenoble 3) y Antonio Romano (Università di Torino). Este último es el coordinador general en la actualidad.

${ }^{2}$ AMPER-Col está coordinado por Mercedes Amparo Muñetón Ayala, profesora de la Universidad de Antioquia, Medellín, Colombia; la sub-coordinadora es Josefa Dorta Luis, profesora de la Universidad de La Laguna, Tenerife, Islas Canarias, España. 
dellín sin estudios superiores. El objetivo principal es comprobar si hay un patrón de asociación entre los parámetros acústicos que permita inferir que en la constitución prosódica de la frase, los tres parámetros son responsables del contorno entonativo más que inferir que uno prima sobre el otro.

La importancia que cada uno de estos parámetros tiene sobre la estructura prosódica del español no es un problema reciente, pero sí actual como lo ponen de manifiesto algunos de los estudios que se han llevado a cabo tanto en la palabra aislada (ver Candia, Urrutia y Fernández, 2006; Llisterri, Machuca, De la Mota, Riera y Ríos, 2005; Mora, 1998; Urrutia, 2007; entre otros) como en contexto (Garrido, Llisterri, De la Mota y Ríos, 1995; Méndez, 2010; Valiente, 2012; entre otros). Sin embargo, en lo que parece haber un acuerdo es que el acento de las palabras aisladas se somete a la prosodia de la frase (Martínez Celdrán y Fernández Planas, 2013: 199). Por tal motivo, los resultados encontrados en las palabras aisladas no se pueden extrapolar a la frase en general.

En el nivel de la palabra, por ejemplo, Cuervo (1954) y Navarro Tomás (1963) sugieren que el correlato del acento es la intensidad; Canellada y Madsen (1987) que es la duración; y Bolinger y Hodapp (1961) y Quilis (1988) que es la F0. Además, algunos autores sugieren que los tres parámetros son importantes para el acento, aunque establecen un orden de relevancia diferente, así, para Contreras (1963) la importancia de la F0 está en primer lugar, la intensidad va en segundo y la duración en tercer lugar; Cabrera (1995) está de acuerdo en la importancia de la F0, pero invierte el orden de los otros dos parámetros, sugiriendo que la duración va en segundo lugar y la intensidad en tercer lugar.

Por su parte, en la prosodia de la frase, estructura en la que se enmarca el presente trabajo, también existe controversia. Por ejemplo, Candia et al. (2006) sugieren que la F0, la duración e intensidad son relevantes en la configuración prosódica de la frase. Los autores llevaron a cabo un experimento en el que participaron cuatro estudiantes universitarios del norte de España (dos mujeres y dos hombres), quienes leyeron en voz alta 72 enunciados no conectados entre sí. El objetivo del estudio era medir los valores de cuatro variables acústicas: el tono, la amplitud, la duración de la vocal y la duración de la sílaba. También, se incluyó una variable que marca la condición acentual de la sílaba: lexicalmente acentuada, pre-acentuada o post-acentuada. De esta forma, los autores querían responder a la pregunta de si la sílaba acentuada era la que mayor intensidad, tono o duración tenía dentro de la secuencia léxica en la que aparecía. Para ello, llevaron a cabo análisis estadísticos de correlación parcial y de regresión múltiple que mostraron una alta correlación e interacción entre los tres parámetros.

No obstante, Garrido et al. (1995), en un estudio realizado con anterioridad encontraron resultados diferentes. Los investigadores compararon las características prosódicas de cinco oraciones (cada una de 15 sílabas). Éstas fueron leídas de forma aislada y contextualizadas en párrafos por cinco estudiantes universitarios, 
hablantes masculinos del español estándar. Una de las conclusiones indica que la duración es un correlato importante en la caracterización del acento léxico puesto que las sílabas tónicas presentan diferencias estadísticamente significativas con las átonas. Con el fin de analizar la correlación entre la duración y la F0, los investigadores llevaron a cabo la correlación de Pearson, a diferencia del estudio de Candia et al. (2006), en este estudio no encontraron correlación entre los parámetros de F0 y duración en ningún tipo de sílaba.

Más recientemente, Méndez (2010), llevó a cabo un estudio en el habla venezolana andina en el que muestra relación entre los parámetros acústicos de frecuencia fundamental y de duración. En este estudio se utilizó el corpus fijo de las oraciones declarativas e interrogativas del proyecto AMPER-VE ${ }^{3}$. Los participantes fueron cuatro mujeres sin estudios superiores, dos de la zona urbana y dos de la zona rural. Se realizó un análisis descriptivo de cada una de las variables y el autor llegó a la conclusión de que hay una relación inversa entre el campo tonal y el campo durativo, esto es, en las frases declarativas un menor campo tonal interactúa con un mayor campo durativo, dándose el patrón inverso en las interrogativas.

Valiente (2012), por su parte, realizó un estudio con el objetivo de determinar las correlaciones entre la F0 y la duración en el habla informal en la modalidad enunciativa. Para ello, llevó a cabo el análisis de correlación de Pearson en un corpus conformado por 42 grupos tónicos de cuatro hombres y cuatro mujeres de la comarca de la Hurdes, Extremadura. Los resultados mostraron que no existe una relación lineal entre la F0 y la duración.

Muñetón (2016) realizó, en el marco del proyecto AMPER-Col, un trabajo con el objetivo de analizar el comportamiento de la frecuencia fundamental, la duración y la intensidad en oraciones sin expansión, con expansión en el sujeto y con expansión en el objeto, en la modalidad interrogativa en el habla de un hombre urbano sin estudios de Medellín. Los análisis los basó en la correlación de Pearson tomando los datos absolutos de las tres variables. Los resultados revelaron que a diferencia de las pretónicas y postónicas las tres variables correlacionan en la tónica de la siguiente manera: la duración correlaciona de forma negativa con la F0 y la intensidad, mientras que estas dos últimas variables lo hacen de forma positiva. Además, el estudio muestra que la tónica tiene una mayor duración que las vocales adyacentes, por tal motivo, los datos permitieron inferir que a mayor duración menor F0 y menor intensidad.

Desde este punto de vista, se observa que hay diferentes posturas en relación a la prosodia de las frases. Sin embargo, para llegar a estas conclusiones, los investigadores han usado métodos diferentes. Por ejemplo, el análisis descriptivo de cada parámetro (Méndez, 2010) o el uso, específicamente, de la medida de relación lineal de Pearson con el fin de obtener correlaciones entre los parámetros (Candia

${ }^{3}$ AMPER-VE $=$ Proyecto AMPER Venezuela. 
et al., 2006; Garrido et al., 1995; Muñetón, 2016 y Valiente, 2012). A diferencia de los anteriores estudios, en el presente se va a usar el análisis estadístico de tablas de contingencia aplicando la prueba de chi al cuadrado de Pearson. Se ha escogido esta prueba porque permite conocer dos datos relevantes para responder al problema que se aborda: 1) la fuerza de asociación existente entre los parámetros acústicos y 2) si esta fuerza de asociación está por encima del azar o no.

Por otra parte, los diferentes estudios han usado valores absolutos en los análisis, esto es, valores directos que no han sido normalizados. Sin embargo, el uso de valores relativos es ventajoso porque consiste en detectar las diferencias mínimas perceptibles (DMP) por el oído humano puesto que "si el umbral funcional fuese idéntico al umbral perceptivo, habría un excesivo riesgo de pérdida de información, al exigir que la capacidad perceptiva del oyente esté trabajando siempre al máximo de sus posibilidades físicas" (Pamies Bertrán, Fernández Planas, Martínez Celdrán, Ortega Escandell y Amorós Céspedes, 2001). De esta manera, con el uso de valores relativos se aíslan aquellas diferencias mínimas funcionales de F0, duración e intensidad que son relevantes para el oído humano en la conformación prosódica.

Respecto a las DMP hay diferentes propuestas para el análisis de la F0 (ver Marrero, 2008; Pamies Bertrán et al., 2001) y la duración (ver Marrero, 2008; Pamies Bertrán y Fernández Planas, 2006) dependiendo del experimento realizado o de la lengua, en cambio las investigaciones sobre la intensidad son menos frecuentes, aunque al respecto se han hecho algunas propuestas (ver Marrero, 2008; Muñetón, 2016). Por tal motivo, en el presente trabajo, en el análisis de los tres parámetros prosódicos se ha tenido en cuenta la literatura actual sobre las DMP en español. En el análisis de la F0 Pamies Bertrán et al. (2001) establecieron un umbral de 1,5 semitonos ${ }^{4}$. Ellos diseñaron un test de percepción usando una tarea de igual/diferente. El test contenía una sola sílaba a la cual se le modificó la frecuencia hasta generar 16 pares de sílabas (ba-ba) que tonalmente diferían entre sí y 6 pares que eran isotonales. La tarea del participante consistía en decir si las sílabas las percibía como iguales o diferentes. Los resultados obtenidos coinciden con lo propuesto por Rietveld y Gussenhoven (1985) en un estudio realizado en holandés y, también, con otro realizado en español por Toledo (2000). En el análisis de la duración Pamies Bertrán y Fernández Planas (2006) propusieron un umbral del 36\%, el cual es muy similar a la diferencia mínima en música (33\%). Los investigadores llevaron a cabo un test de percepción con una tarea de igual/ diferente de forma similar que en el estudio de Pamies Bertrán et al. (2001), sin embargo, en esta ocasión usaron la sílaba /mam/. Además, había ocho pares con una duración diferente y un par con una duración igual.

Para el análisis de la intensidad Martín (2010) sugirió un umbral de +-3 dB.

${ }^{4} \mathrm{La}$ fórmula para hallar la distancia tonal entre dos frecuencias en valores absolutos es $\mathrm{D}=((12 /$ $\left.\operatorname{LOG}(2))^{*}(\operatorname{LOG}(\mathrm{B} 2 / \mathrm{B} 3))\right)$ (Nooteboom, 1997). 
El investigador diseñó un procedimiento teniendo en cuenta la fluctuación de la intensidad debido al cansancio del hablante o la distancia de éste con el micrófono. Esta propuesta la hizo con el fin de relativizar los valores de intensidad de 10 hablantes que provenían de Madrid y otros tantos de Tenerife. El procedimiento para establecer el umbral perceptivo de la intensidad consistió en 1) hallar la intensidad de cada una de las vocales de la oración; 2) obtener la media de la oración; 3 ) buscar la diferencia entre la media de la oración y el valor de cada vocal; y 4) restar la diferencia obtenida a un valor estándar de $20 \mathrm{~dB}^{5}$, con el fin de obtener el valor relativo de cada vocal. Si este valor es igual o mayor a $+-3 \mathrm{~dB}$ se considera que hay DMP entre las dos vocales.

\section{OBJETIVO}

Teniendo como base lo anterior, el presente estudio explora las ocurrencias de la F0, duración e intensidad en oraciones declarativas e interrogativas en el habla de una mujer perteneciente a la zona urbana de Medellín sin estudios superiores. Para ello, se usa el análisis estadístico de tablas de contingencias con el fin de observar la fuerza de asociación entre los diferentes parámetros con base en las DMP. Desde esta perspectiva, el presente trabajo se encuadra en el debate de si el contorno entonativo de la frase se caracteriza por tener un solo correlato prosódico o si éstos muestran un patrón de coocurrencia. Si los datos muestran que los parámetros no están asociados por encima del azar indicarían que uno de ellos es el correlato del contorno melódico, si por el contrario sí coocurren indicarían que en la conformación prosódica de la frase intervienen los tres parámetros.

En este sentido, las preguntas de investigación son dos: la primera se enfoca a analizar si hay o no asociación entre los tres parámetros prosódicos; la segunda se refiere a si la existencia o no de estas asociaciones establecen diferencias entre las oraciones declarativas y las interrogativas.

\section{METODOLOGÍA}

\subsection{Participante y procedimiento}

En este trabajo se siguió la metodología establecida en el proyecto AMPER (ver Martínez Celdrán y Fernández Planas, 2006). Dado que los estudios de entonación colombiana en el marco del proyecto se están iniciando, este estudio prelimi-

${ }^{5}$ Se toma este valor porque en una misma frase las diferencias de intensidad entre las vocales oscilan alrededor de 20 dB (Dorta, Díaz, Hernández, Jorge y Martín, 2013:83). 
nar analiza las emisiones de una mujer sin estudios de la zona urbana de Medellín, su edad está comprendida entre los 25 y 45 años. El material analizado se compone de 378 oraciones, 189 declarativas y otras tantas interrogativas tomadas del corpus fijo del proyecto AMPER-COL. Cada una de las oraciones se repitió tres veces, a continuación se extrajo la media de las tres repeticiones del corpus. Dentro de estas oraciones, nueve pertenecen a las oraciones sin expansión $(\mathrm{S}+\mathrm{V}+\mathrm{O})$, otras 27 a las oraciones con expansión en el sujeto $(\mathrm{S}+\mathrm{EXP}+\mathrm{V}+\mathrm{O})$ y otras 27 a las oraciones con expansión en el objeto $(\mathrm{S}+\mathrm{V}+\mathrm{O}+\mathrm{EXP})$. El sintagma inicial y el final como la expansión del sujeto o del objeto están compuestos por trisílabos de igual tipología acentual en el interior de cada oración (oxítona, paroxítona, proparoxítona); el sintagma central permanece invariable (acento paroxítono). Se trata de frases del tipo El saxofón se toca con emoción. La guitarra clásica se toca con paciencia. La máquina se toca con pánico práctico.

\subsection{Análisis acústico}

Las grabaciones se hicieron en la cabina insonorizada del laboratorio de fonética de la Universidad de Antioquia. Se utilizó el programa Goldwave 4.25 para el tratamiento de los ficheros de sonido (.wav) y éstos fueron analizados posteriormente con el programa AMPER-2006 (López, Muñiz, Díaz, Corral, Brezmes y Alvarellos, 2007) en el entorno Matlab Matriz Laboratory ${ }^{6}$. Las rutinas creadas en Matlab permiten obtener, a partir de la segmentación en el oscilograma, tres valores de F0 de cada vocal silábica (en el inicio, en el medio y en el final), el valor de la duración y de la intensidad.

\subsection{Codificación de los datos}

La codificación de los datos se llevó a cabo en cinco pasos. En el primero se segmentaron las oraciones en tres grupos sintagmáticos: SN (sintagma nominal), SV (sintagma verbal) y SP (sintagma preposicional) en cada una de las modalidades. En el segundo se extrajeron los valores de cada parámetro acústico: la F0 medial (medida en $\mathrm{Hz}$ ); la duración (medida en ms) y la intensidad (medida en dB) de cada uno de los segmentos vocálicos. En el tercero se rotularon cada una de las vocales como pretónica, tónica o postónica, sea esta última por fonética normal o fonética sintáctica. Esto es, si, por ejemplo, hay que rotular la secuencia "el bandolín se toca" la "e" de la sílaba "se" es la postónica de la tónica "lín".

En el cuarto paso se identificaron las DMP existentes en el contraste entre

\footnotetext{
${ }^{6}$ Licencia No 878004 del laboratorio de fonética de la Universidad de Antioquia.
} 
la pretónica-tónica (Pr-T) y la tónica-la postónica (T-Ps). Este cálculo se hizo en cada uno de los tres parámetros prosódicos. Así, el umbral perceptivo de la F0 se estableció en 1,5 St, según lo propuesto por Pamies Bertrán et al. (2001). El umbral de la duración se fijó en el 33.33\% teniendo como base lo propuesto por Fernández Planas y Martínez Celdrán (2003). Finalmente, el umbral de la intensidad se estableció en $+-3 \mathrm{~dB}$, acogiendo la propuesta de Martín (2010). Si la diferencia entre las dos vocales comparadas era igual o superior al umbral establecido se consideraba que había DMP y se categorizaba como 1, de otra forma se categorizaba como 0.

En el quinto paso se usaron tablas de contingencia con el fin de examinar si las diferencias mínimas identificadas en cada uno de los parámetros presentaban asociaciones y si éstas estaban por encima de lo esperado por azar. Se llevaron a cabo los análisis usando chi al cuadrado, cruzando los tres parámetros por pares. Se usaron los residuos corregidos tipificados con el fin de identificar celdas en las cuales las puntuaciones $\mathrm{Z}$ fueran iguales o mayores a 1.96, de forma positiva o negativa (Haberman, 1973). Una puntuación positiva indica que la frecuencia de la celda es mayor de lo que cabría esperar por azar, mientras que una puntuación negativa significa que la frecuencia es menor de lo que cabría esperar por azar.

\section{RESULTADOS}

Los resultados se presentan en dos apartados: en el primero (4.2) se describe la distribución de las DMP en los diferentes parámetros acústicos y en el segundo (4.3) se analizan las asociaciones de los mismos por medio del estudio de las tablas de contingencia. En ambos apartados se verán los resultados específicos a cada modalidad oracional.

\subsection{Datos generales}

Tomando las oraciones en conjunto, el total de vocales asciende a 1.722, de las cuales el $50 \%$ pertenece a cada modalidad. Las vocales tónicas corresponden a 243 y las átonas a 309 tanto en la modalidad declarativa como en la interrogativa.

\subsection{Descripción de las DMP en los diferentes parámetros acústicos}

Las Tablas I, II y III muestran la comparación entre la modalidad declarativa e interrogativa de los casos que presentan diferencias significativas entre la $\mathrm{Pr}-\mathrm{T}$ y de T-Ps en función de los diferentes parámetros de F0, duración e intensidad. 
Específicamente, en la frecuencia fundamental hay 510 casos que presentan DMP, de los cuales el $42.71 \%$ pertenecen a la modalidad declarativa y el $57.26 \%$ a la modalidad interrogativa. En la duración se encontraron 480 casos, de los cuales el $55.81 \%$ corresponde a la modalidad declarativa y el $44.19 \%$ a la modalidad interrogativa. Finalmente, en la intensidad se hallaron 277 casos, de los cuales el 51.30 $\%$ pertenecen a la modalidad declarativa y el $48.70 \%$ a la modalidad interrogativa.

En términos generales, los datos indican que la frecuencia fundamental es el parámetro que presenta un porcentaje mayor de DMP en comparación a la duración y a la intensidad. En segundo lugar está el parámetro de duración. Por otra parte, la intensidad y la duración presentan una distribución de casos similar en ambas modalidades, en contraste con el parámetro de F0 en el cual las oraciones interrogativas presentan un número de casos superior que las declarativas. A continuación se analizan con detenimiento cada uno de los parámetros en función de los contrastes entre Pr-T y T-Ps.

Tabla I. Comparación entre la modalidad declarativa e interrogativa de los casos (entre paréntesis el porcentaje) con DMP entre la Pr-T y de T-Ps en función de la F0.

\begin{tabular}{|c|c|c|c|c|c|c|c|c|c|c|c|c|c|}
\hline & \multicolumn{6}{|c|}{ Modalidad declarativa } & \multicolumn{7}{|c|}{ Modalidad interrogativa } \\
\hline & \multicolumn{3}{|c|}{ Pretónica-Tónica } & \multicolumn{3}{|c|}{ Tónica - Postónica } & \multicolumn{3}{|c|}{ Pretónica-Tónica } & \multicolumn{4}{|c|}{ Tónica - Postónica } \\
\hline & $\operatorname{Pr}$ & $\mathrm{T}$ & $\begin{array}{l}\text { Total } \\
\text { Pr }-\mathrm{T}\end{array}$ & $\mathrm{T}$ & Ps & $\begin{array}{l}\text { Total } \\
\text { T-Ps }\end{array}$ & $\operatorname{Pr}$ & $\mathrm{T}$ & $\begin{array}{l}\text { Total } \\
\text { Pr-T }\end{array}$ & $\mathrm{T}$ & Ps & $\begin{array}{l}\text { Total } \\
\text { T-Ps }\end{array}$ & Total \\
\hline \multicolumn{14}{|l|}{ F0 } \\
\hline $\mathrm{SN}$ & $\begin{array}{c}8 \\
(1.57)\end{array}$ & $\begin{array}{c}36 \\
(7.06)\end{array}$ & $\begin{array}{c}44 \\
(8.63)\end{array}$ & -- & $\begin{array}{c}54 \\
(10.58)\end{array}$ & $\begin{array}{l}54 \\
(10.58)\end{array}$ & $\begin{array}{c}36 \\
(7.05)\end{array}$ & $\begin{array}{c}2 \\
(0.40)\end{array}$ & $\begin{array}{l}38 \\
(7.45)\end{array}$ & -- & $\begin{array}{c}71 \\
(14.00)\end{array}$ & $\begin{array}{l}71 \\
(14)\end{array}$ & $\begin{array}{c}207 \\
(40.21)\end{array}$ \\
\hline SV & $\begin{array}{c}60 \\
(11.76)\end{array}$ & -- & $\begin{array}{c}60 \\
(11.76)\end{array}$ & -- & $\begin{array}{c}7 \\
(1.37)\end{array}$ & $\begin{array}{l}7 \\
(1.37)\end{array}$ & $\begin{array}{c}41 \\
(8.03)\end{array}$ & -- & $\begin{array}{l}41 \\
(8.03)\end{array}$ & -- & $\begin{array}{c}34 \\
(6.70)\end{array}$ & $\begin{array}{l}34 \\
(6.70)\end{array}$ & $\begin{array}{c}142 \\
(27.84)\end{array}$ \\
\hline SP & $\begin{array}{c}27 \\
(5.29)\end{array}$ & $\begin{array}{c}9 \\
(1.76)\end{array}$ & $\begin{array}{c}36 \\
(7.05)\end{array}$ & $\begin{array}{c}8 \\
(1.56)\end{array}$ & $\begin{array}{c}9 \\
(1.76)\end{array}$ & $\begin{array}{l}17 \\
(3.32)\end{array}$ & $\begin{array}{c}6 \\
(1.17)\end{array}$ & $\begin{array}{c}52 \\
(10.19)\end{array}$ & $\begin{array}{l}58 \\
(11.36)\end{array}$ & $\begin{array}{c}37 \\
(7.25)\end{array}$ & $\begin{array}{c}13 \\
(2.50)\end{array}$ & $\begin{array}{l}50 \\
(9.75)\end{array}$ & $\begin{array}{c}161 \\
(31.56)\end{array}$ \\
\hline Total & $\begin{array}{c}95 \\
(18.62)\end{array}$ & $\begin{array}{c}45 \\
(8.82)\end{array}$ & $\begin{array}{c}140 \\
(27.44)\end{array}$ & $\begin{array}{c}8 \\
(1.56)\end{array}$ & $\begin{array}{c}70 \\
(13.72)\end{array}$ & $\begin{array}{l}78 \\
(15.27)\end{array}$ & $\begin{array}{c}83 \\
(16.27)\end{array}$ & $\begin{array}{c}54 \\
(10.59)\end{array}$ & $\begin{array}{l}137 \\
(26.84)\end{array}$ & $\begin{array}{c}37 \\
(7.25)\end{array}$ & $\begin{array}{c}118 \\
(23.20)\end{array}$ & $\begin{array}{l}155 \\
(30.45)\end{array}$ & $\begin{array}{c}510 \\
(100)\end{array}$ \\
\hline
\end{tabular}

Abreviaturas: Pr: pretónica; T: tónica; Ps: postónica; SN: sintagma nominal; SV: Sintagma verbal; SP: sintagma preposicional.

\subsubsection{La frecuencia fundamental}

En la Tabla I se presentan los datos de la frecuencia fundamental. En relación al contraste entre Pr-T se observó que hay una distribución de DMP similar en ambas modalidades (declarativas 27,44\%; interrogativas 26,84\%) y, además, que recae un porcentaje mayor de DMP en las pretónicas (D: 18.62\%; I: 16.27\%) que 
en las tónicas (D: 8.82\%; I: 10.59\%). No obstante, al analizar cada uno de los sintagmas se encontró que en este contraste ambas modalidades presentan patrones inversos. Es decir, en el SN de las oraciones declarativas el mayor porcentaje de DMP recae en las tónicas (7.06\%), mientras que en las interrogativas el mayor porcentaje recae en las pretónicas $(7.05 \%)$. En el SP se presenta un patrón diferente, esto es, en las oraciones declarativas el mayor porcentaje recae en las pretónicas $(5.29 \%)$ mientras que en las interrogativas recae en las tónicas (10.19\%).

En cuanto al contraste entre la T-Ps, la modalidad interrogativa duplica el porcentaje de la modalidad declarativa (D: 15,27\%; I: 30,45\%). Sin embargo, en ambas modalidades el mayor porcentaje recae en la postónica (D: 13.72\%; I: 23.20\%). Además, el SN exhibe el porcentaje mayor de DMP, las cuales recaen en su totalidad en la postónica en ambas modalidades (D:10.58; I: 14\%). El SP continúa en el número de distribución de DMP, sin embargo las oraciones interrogativas tienen un porcentaje superior en la tónica $(7.25 \%)$ que en la postónica (2.50\%), mientras que en las declarativas las diferencias entre la tónica $(1.56 \%)$ y la postónica $(1.76 \%)$ es leve.

Estos datos preliminares señalan diferencias entre la modalidad declarativa e interrogativa. Así, en el contraste $\operatorname{Pr}-\mathrm{T}$, ambas modalidades presentan un patrón inverso en el SN y en el SP. Mientras que en el SN el mayor porcentaje recae en las tónicas de las declarativas, en las interrogativas recae en las pretónicas. Asimismo, en el SP el mayor porcentaje recae en las tónicas de las oraciones declarativas, en las interrogativas recae en las pretónicas. En el otro contraste, esto es, T-Ps la diferencia básica entre ambas modalidades radica en que las tónicas presentan un número superior de DMP que las postónicas en las interrogativas mientras que en las declarativas ambas vocales presentan un número similar de DMP.

\subsubsection{La duración}

En la Tabla II se presentan los datos de la duración. Respecto al contraste entre $\operatorname{Pr}-\mathrm{T}$, hay un porcentaje mayor de casos en la modalidad declarativa $(26.66 \%)$ que en la interrogativa (20.82), sin embargo, en el $100 \%$ de los casos con DMP la mayor duración coincide con la tónica. El porcentaje mayor de DMP recae en el SP en ambas modalidades (D: 13.95\%; I: 11,25\%), y en segundo lugar en el SN (D: $8.75 \%$; I: 6.87\%).

En relación al contraste T-Ps, se presenta un patrón similar al ya mencionado, es decir, las oraciones declarativas $(29,16 \%)$ muestran un porcentaje mayor que las interrogativas (23,37\%), y la mayor duración recae siempre en las tónicas. Así mismo, el SP exhibe el porcentaje más alto de DMP (D: 14.58\%; I: 12.34\%) y, a continuación, está el SN (D: 13.75\%; I: 10.83\%) independientemente de la modalidad.

Por tanto, tomando los datos en conjunto, la modalidad declarativa y la interrogativa presentan un patrón similar en este parámetro acústico. 
Tabla II. Comparación entre la modalidad declarativa e interrogativa de los casos (entre paréntesis el porcentaje) con DMP entre la Pr-T y de T-Ps en función de la duración.

\begin{tabular}{|c|c|c|c|c|c|c|c|c|c|c|c|c|c|}
\hline & \multicolumn{6}{|c|}{ Modalidad declarativa } & \multicolumn{6}{|c|}{ Modalidad interrogativa } & \\
\hline & \multicolumn{3}{|c|}{ Pretónica-Tónica } & \multicolumn{3}{|c|}{ Tónica - Postónica } & \multicolumn{3}{|c|}{ Pretónica-Tónica } & \multicolumn{4}{|c|}{ Tónica - Postónica } \\
\hline & $\operatorname{Pr}$ & $\mathrm{T}$ & $\begin{array}{l}\text { Total } \\
\text { Pr -T }\end{array}$ & $\mathrm{T}$ & Ps & $\begin{array}{l}\text { Total } \\
\text { T-Ps }\end{array}$ & $\operatorname{Pr}$ & $\mathrm{T}$ & $\begin{array}{l}\text { Total } \\
\text { Pr-T }\end{array}$ & $\mathrm{T}$ & Ps & $\begin{array}{l}\text { Total } \\
\text { T-Ps }\end{array}$ & Total \\
\hline \multicolumn{14}{|c|}{ Duración } \\
\hline SN & -- & $\begin{array}{c}42 \\
(8.75)\end{array}$ & $\begin{array}{c}42 \\
(8.75)\end{array}$ & $\begin{array}{c}66 \\
(13.75)\end{array}$ & -- & $\begin{array}{l}66 \\
(13.75)\end{array}$ & -- & $\begin{array}{c}33 \\
(6.87)\end{array}$ & $\begin{array}{l}33 \\
(6.87)\end{array}$ & $\begin{array}{c}52 \\
(10.83)\end{array}$ & -- & $\begin{array}{l}52 \\
(10.83)\end{array}$ & $\begin{array}{c}193 \\
(40.20)\end{array}$ \\
\hline SV & -- & $\begin{array}{c}19 \\
(3.95)\end{array}$ & $\begin{array}{c}19 \\
(3.95)\end{array}$ & $\begin{array}{c}4 \\
(0.83)\end{array}$ & -- & $\begin{array}{l}4 \\
(0.83)\end{array}$ & -- & $\begin{array}{c}13 \\
(2.70)\end{array}$ & $\begin{array}{l}13 \\
(2.70)\end{array}$ & $\begin{array}{c}1 \\
(0.20)\end{array}$ & - & $\begin{array}{l}1 \\
(0.20)\end{array}$ & $\begin{array}{c}37 \\
(7.68)\end{array}$ \\
\hline SP & -- & $\begin{array}{c}67 \\
(13.95)\end{array}$ & $\begin{array}{c}67 \\
(13.95)\end{array}$ & $\begin{array}{c}70 \\
(14.58)\end{array}$ & -- & $\begin{array}{l}70 \\
(14.58)\end{array}$ & -- & $\begin{array}{c}54 \\
(11.25)\end{array}$ & $\begin{array}{l}54 \\
(11.25)\end{array}$ & $\begin{array}{c}59 \\
(12.34)\end{array}$ & - & $\begin{array}{l}59 \\
(12.34)\end{array}$ & $\begin{array}{c}250 \\
(52.12)\end{array}$ \\
\hline Total & -- & $\begin{array}{c}128 \\
(26.66)\end{array}$ & $\begin{array}{c}128 \\
(26.66)\end{array}$ & $\begin{array}{c}140 \\
(29.16)\end{array}$ & -- & $\begin{array}{l}140 \\
(29.16)\end{array}$ & -- & $\begin{array}{c}100 \\
(20.83)\end{array}$ & $\begin{array}{l}100 \\
(20.82)\end{array}$ & $\begin{array}{c}112 \\
(23.33)\end{array}$ & - & $\begin{array}{l}112 \\
(23.37)\end{array}$ & $\begin{array}{c}480 \\
(100)\end{array}$ \\
\hline
\end{tabular}

\subsubsection{La intensidad}

En la Tabla III se presentan los datos de la intensidad. En el contraste Pr-T, las DMP de la modalidad interrogativa $(22.73 \%)$ muestran un porcentaje levemente superior a la modalidad declarativa (20.21\%) siendo la vocal tónica (D: 11.18\%; I: $13.35 \%$ ) en la que recae una proporción mayor de DMP. El mayor porcentaje de DMP recae en el SN (D: 12.27\%; I: 15.88\%), sin embargo, en las oraciones declarativas la mayor proporción recae en la pretónica $(6.50 \%)$ mientras que en las interrogativas recae en la tónica $(9.39 \%)$.

En el contraste T-Ps, la modalidad declarativa $(31,07 \%)$ presenta un porcentaje mayor de casos con DMP que la modalidad interrogativa (25.99\%), sin embargo, en ambas modalidades el mayor porcentaje recae en la tónica (D: $23.10 \%$; I: 19.86\%). Asimismo, es el SP en el que se da una mayor concentración de DMP (D: $16.61 \%$; I: $15.52 \%)$. Por tanto, en ambos contrastes y en ambas modalidades las vocales tónicas presentan una distribución mayor de DMP que las vocales adyacentes. El SV no presenta DMP en ninguna de las modalidades ni en ninguno de los contrastes.

En definitiva, los datos precedentes indican que las diferencias entre ambas modalidades parecen estar en la modulación de la F0, como ya se explicó, porque en los otros dos parámetros los porcentajes de DMP son equivalentes.

Una vez descritos estos parámetros se pasó a observar las asociaciones entre ellos a través de los análisis de contingencias. 
Tabla III. Comparación entre la modalidad declarativa e interrogativa de los casos (entre paréntesis el porcentaje) con DMP entre la Pr-T y de T-Ps en función de la intensidad.

\begin{tabular}{|c|c|c|c|c|c|c|c|c|c|c|c|c|c|}
\hline & \multicolumn{6}{|c|}{ Modalidad declarativa } & \multicolumn{7}{|c|}{ Modalidad interrogativa } \\
\hline & \multicolumn{3}{|c|}{ Pretónica-Tónica } & \multicolumn{3}{|c|}{ Tónica - Postónica } & \multicolumn{3}{|c|}{ Pretónica-Tónica } & \multicolumn{4}{|c|}{ Tónica - Postónica } \\
\hline & $\operatorname{Pr}$ & $\mathrm{T}$ & $\begin{array}{l}\text { Total } \\
\text { Pr -T }\end{array}$ & $\mathrm{T}$ & Ps & $\begin{array}{l}\text { Total } \\
\text { T-Ps }\end{array}$ & $\operatorname{Pr}$ & $\mathrm{T}$ & $\begin{array}{l}\text { Total } \\
\text { Pr-T }\end{array}$ & $\mathrm{T}$ & Ps & $\begin{array}{l}\text { Total } \\
\text { T-Ps }\end{array}$ & Total \\
\hline \multicolumn{14}{|c|}{ Intensidad } \\
\hline $\mathrm{SN}$ & $\begin{array}{c}18 \\
(6.50)\end{array}$ & $\begin{array}{c}16 \\
(5.78)\end{array}$ & $\begin{array}{c}34 \\
(12.27)\end{array}$ & $\begin{array}{c}7 \\
(2.53)\end{array}$ & $\begin{array}{c}21 \\
(7.60)\end{array}$ & $\begin{array}{l}28 \\
(10.13)\end{array}$ & $\begin{array}{c}18 \\
(6.50)\end{array}$ & $\begin{array}{c}26 \\
(9.39)\end{array}$ & $\begin{array}{l}44 \\
(15.88)\end{array}$ & $\begin{array}{c}11 \\
(3.97)\end{array}$ & $\begin{array}{c}14 \\
(5.05)\end{array}$ & $\begin{array}{l}25 \\
(9.03)\end{array}$ & $\begin{array}{c}131 \\
(47.30)\end{array}$ \\
\hline SV & -- & $\begin{array}{c}9 \\
(3.24)\end{array}$ & $\begin{array}{c}9 \\
(3.24)\end{array}$ & $\begin{array}{c}12 \\
(4.33)\end{array}$ & -- & $\begin{array}{l}12 \\
(4.33)\end{array}$ & -.. & $\begin{array}{c}9 \\
(3.24)\end{array}$ & $\begin{array}{l}9 \\
(3.24)\end{array}$ & $\begin{array}{c}4 \\
(1.44)\end{array}$ & -- & $\begin{array}{l}4 \\
(1.44)\end{array}$ & $\begin{array}{c}34 \\
(12.25)\end{array}$ \\
\hline SP & $\begin{array}{c}7 \\
(2.53)\end{array}$ & $\begin{array}{c}6 \\
(2.17)\end{array}$ & $\begin{array}{c}13 \\
(4.69)\end{array}$ & $\begin{array}{c}45 \\
(16.25)\end{array}$ & $\begin{array}{c}1 \\
(0.36)\end{array}$ & $\begin{array}{l}46 \\
(16.61)\end{array}$ & $\begin{array}{c}8 \\
(2.89)\end{array}$ & $\begin{array}{c}2 \\
(0.72)\end{array}$ & $\begin{array}{l}10 \\
(3.61)\end{array}$ & $\begin{array}{c}40 \\
(14.44)\end{array}$ & $\begin{array}{c}3 \\
(1.08)\end{array}$ & $\begin{array}{l}43 \\
(15.52)\end{array}$ & $\begin{array}{c}112 \\
(40.43)\end{array}$ \\
\hline Total & $\begin{array}{c}24 \\
(9.02)\end{array}$ & $\begin{array}{c}31 \\
(11.18)\end{array}$ & $\begin{array}{c}56 \\
(20.21)\end{array}$ & $\begin{array}{c}64 \\
(23.10)\end{array}$ & $\begin{array}{c}22 \\
(7.96)\end{array}$ & $\begin{array}{l}86 \\
(31.07)\end{array}$ & $\begin{array}{c}26 \\
(9.39)\end{array}$ & $\begin{array}{c}37 \\
(13.35)\end{array}$ & $\begin{array}{l}63 \\
(22.73)\end{array}$ & $\begin{array}{c}55 \\
(19.86)\end{array}$ & $\begin{array}{c}17 \\
(6.14)\end{array}$ & $\begin{array}{l}72 \\
(25.99)\end{array}$ & $\begin{array}{c}277 \\
(100)\end{array}$ \\
\hline
\end{tabular}

\subsection{Asociación de los parámetros acústicos}

En primer lugar se muestran los resultados de las contingencias de la F0 y la duración; de la F0 y la intensidad; y, finalmente, de la intensidad y la duración en los diferentes sintagmas de las oraciones declarativas e interrogativas teniendo como base las DMP de los contrastes entre Pr-T y entre T-Ps.

En forma general, los resultados muestran que el 100\% de contingencias encontradas en los análisis son positivas, lo que indica que los parámetros acústicos coocurren en el contorno entonativo. De estas contingencias, el 64.28\% coocurren en la misma sílaba ( $77.77 \%$ en las tónicas, $11.11 \%$ en las pretónicas y el otro tanto en las postónicas), y en el $35.72 \%$ restante las asociaciones se presentan en sílabas diferentes en las contingencias entre F0-duración y F0-intensidad.

\subsubsection{Fo y duración}

Sintagma nominal. Las contingencias entre la frecuencia fundamental y la duración fueron estadísticamente significativas en el contraste $\operatorname{Pr}-\mathrm{T}$ en las oraciones declarativas $\left(\chi^{2}(2, n=23)=8.41, \mathrm{p}=.015\right)$, y en las oraciones interrogativas $\left(\chi^{2}(2\right.$, $n=21)=10.87, \mathrm{p}=.002)$. Los residuos tipificados $(r)$ muestran asociaciones positivas por encima del azar entre la duración y la F0 en cada una de las modalidades. Específicamente, en las oraciones declarativas la asociación entre los dos parámetros se presentó en las tónicas $\left(r_{z}=2.4\right)$. Por tanto, estas vocales son perceptivamente más largas y con mayor frecuencia que las pretónicas. En las oraciones 
interrogativas las asociaciones se evidenciaron entre la F0 de la pretónica y la duración de la tónica $\left(r_{z}=2.8\right)$. Esto indica que cuando la tónica presenta diferencias perceptivamente significativas con la pretónica en duración, la F0 de la pretónica las presenta en relación a la tónica. Por tal motivo, los resultados muestran que hay una fuerza de asociación entre la F0 y la duración tanto en las oraciones declarativas como interrogativas, aunque no siguen el mismo patrón de asociación.

En la comparación entre la T-Ps, las contingencias entre la frecuencia fundamental y la duración fueron estadísticamente significativas en las oraciones declarativas $\left(\chi^{2}(2, n=46)=14.86, \mathrm{p}=.001\right)$ y en las interrogativas $\left(\chi^{2}(2, n=45)\right.$ $=63.47, \mathrm{p}<.001)$. Los residuos tipificados $\left(r_{z}\right)$ muestran que las asociaciones estuvieron por encima del azar y fueron positivas en ambas modalidades. Concretamente, las coocurrencias se presentaron entre la duración de la tónica y la F0 de la postónica tanto en las oraciones declarativas como interrogativas $\left(r_{z}=3.1, r_{z}=\right.$ 2.8 , respectivamente). Por tanto, se puede inferir que cuando la tónica es perceptivamente más larga que la postónica, esta última presenta una F0 perceptivamente mayor que la tónica.

Sintagma preposicional. La F0 y la duración en el contraste entre Pr-T fueron estadísticamente significativas en las oraciones interrogativas $\left({ }^{2}(2, n=51)=\right.$ 45.02, $\mathrm{p}<.001)$. Los residuos tipificados $\left(r_{z}=6.6\right)$ muestran que las asociaciones entre la F0 y la duración estuvieron por encima del azar y fueron positivas. Las DMP de duración y F0 coocurren en las tónicas. Indicando que las tónicas tienden a tener una duración y una F0 perceptivamente mayor que las pretónicas en las oraciones interrogativas.

\subsubsection{FO e intensidad}

Sintagma nominal. La frecuencia fundamental y la intensidad fueron estadísticamente significativas en el contraste entre $\operatorname{Pr}-\mathrm{T}$ en las oraciones declarativas $\left(\chi^{2}(2\right.$, $n=7)=25.48, \mathrm{p}<.001)$ y en las interrogativas $\left(\chi^{2}(2, n=16)=35.73, \mathrm{p}<.001\right)$. Los residuos tipificados $\left(r_{z}=4.9, r_{z}=4.2\right.$, declarativas e interrogativas, respectivamente) muestran que las asociaciones entre la F0 y la intensidad estuvieron por encima del azar y fueron positivas. Las asociaciones se presentaron entre la F0 de la pretónica y la intensidad de la tónica en ambas modalidades. Por consiguiente, ambas tipologías oracionales presentan un patrón de asociación similar en el SN. Esto es, cuando la F0 de la pretónica es perceptivamente mayor que la tónica, entonces la tónica tiende a ser perceptivamente más intensa que la pretónica.

El contraste entre la T-Ps de ambos parámetros acústicos indican que hubo diferencias estadísticamente significativas solamente en las oraciones interrogativas $\left(\chi^{2}(2, n=14)=19.95, p=.001\right)$. Los residuos tipificados $\left(r_{z}=2.1\right)$ evidencian que las asociaciones entre la F0 y la intensidad estuvieron por encima del azar y fueron positivas. Además, ambos parámetros coocurren en las postónicas. Por tanto, la 
vocal postónica tiende a ser perceptivamente más intensa y a tener una F0 mayor que la tónica.

Sintagma verbal. La frecuencia fundamental y la intensidad fueron estadísticamente significativas en el rango de comparación entre pretónica y tónica en las oraciones interrogativas $\left(\chi^{2}(1, n=9)=5.25, \mathrm{p}=.022\right)$. Los residuos tipificados $\left(r_{z=}\right.$ 2.3) muestran que las asociaciones entre la F0 y la intensidad estuvieron por encima del azar y fueron positivas. Ambos parámetros coocurren en las pretónicas. Por tanto, ambos parámetros muestran simultáneamente DMP con la tónica en el SV en las oraciones interrogativas.

Sintagma preposicional. La frecuencia fundamental y la intensidad fueron estadísticamente significativas en el contraste entre la $\operatorname{Pr}-\mathrm{T}$ en las oraciones declarativas $\left(\chi^{2}(2, n=6)=10.75, \mathrm{p}=.030\right)$ y en las oraciones interrogativas $\left(\chi^{2}(2, n\right.$ $=8)=10.75, \mathrm{p}=.050)$. Los residuos tipificados $\left(r_{z=} 3.1, r_{z=} 2.4\right.$, declarativas $\mathrm{e}$ interrogativas, respectivamente) muestran que las asociaciones entre la F0 y la intensidad estuvieron por encima del azar y fueron positivas. Las asociaciones de ambos parámetros se presentan en las tónicas de ambas modalidades. Por tanto, las vocales tónicas tienden a tener una F0 y una intensidad perceptivamente mayor que las pretónicas en el SP de ambas modalidades oracionales.

Al observar la F0 y la intensidad en el contraste T-Ps, se observa que fue estadísticamente significativo solamente en las oraciones interrogativas $\left(\chi^{2}(2, n=27)\right.$ $=19.05, \mathrm{p}=.004)$. Los residuos tipificados $\left(r_{z=} 4.5\right)$ muestran que las asociaciones entre la F0 y la intensidad estuvieron por encima del azar y fueron positivas. Además, la asociación de los parámetros se ubica en las vocales tónicas. De esta manera, la F0 y la intensidad de las tónicas presentan simultáneamente DMP en relación a las postónicas en el SP de las oraciones interrogativas.

\subsubsection{Intensidad y duración}

Sintagma nominal. La intensidad y la duración fueron estadísticamente significativas en el contraste Pr-T en las oraciones declarativas $\left(\chi^{2}(2, n=14)=9.93\right.$, $\mathrm{p}=.007)$ y en las oraciones interrogativas $\left(\chi^{2}(2, n=16)=30.12, \mathrm{p}<.001\right)$. Los residuos tipificados $\left(r_{z=} 2.8, r_{z=} 5\right.$, declarativas e interrogativas, respectivamente) muestran que las asociaciones entre la F0 y la intensidad estuvieron por encima del azar y fueron positivas. Las asociaciones se presentaron en las tónicas en ambas modalidades oracionales. Por tanto, la vocal tónica suele ser perceptivamente más larga y más intensa que las pretónicas en el SN de ambas modalidades oracionales.

\section{DISCUSIÓN Y CONCLUSIONES}

Un gran número de lenguas en el mundo emplean el acento como un parámetro 
estructural que especifica cuál sílaba en la palabra es más fuerte que las otras. Uno de los tópicos de la investigación fonética ha sido caracterizar desde una perspectiva acústica las propiedades que identifican las sílabas acentuadas de sus adyacentes. Además, diferentes trabajos han puesto de manifiesto que el acento de la palabra es diferente al usado en la frase. Como se referenció en la introducción, actualmente, existen diferentes propuestas en relación a la asociación entre los tres parámetros basadas en el uso de diferentes métodos de análisis. Por tal motivo, con el fin de ahondar en esta discusión el presente estudio explora las asociaciones de los tres parámetros acústicos en oraciones declarativas e interrogativas en el habla de una mujer urbana de Medellín sin estudios superiores. En primer lugar se observaron las DMP en cada uno de los parámetros prosódicos por ser éstas precisamente las percibidas por el sistema cognitivo. A continuación se realizaron tablas de contingencia con el fin de analizar las asociaciones existentes entre las DMP de los diferentes parámetros y, así, averiguar si existen o no asociaciones entre ellos. Además, se analizó si estas asociaciones establecen diferencias entre las oraciones declarativas y las interrogativas.

En la primera parte del trabajo se describió la distribución de las DMP en los diferentes parámetros prosódicos. El mayor porcentaje se encontró en la F0 y el menor en la intensidad. Respecto a la F0, en términos generales, las oraciones interrogativas, a diferencia de las declarativas, mostraron una mayor proporción de frecuencias. Ahora bien, los datos muestran que el mayor porcentaje de DMP se sincroniza con la sílaba postónica, en segundo lugar con la pretónica y finalmente con la tónica. Por tanto, están en línea con las afirmaciones de Sosa (1999) quien sostiene que "un gran número de picos tonales se corresponden con las sílabas inacentuadas que siguen a la acentuada”. Este fenómeno es conocido como posrrealización $\left(\mathrm{H}^{*} \mathrm{~L}\right)$ del pico tonal y el caso opuesto, esto es, que el pico se sincronice con la pretónica, como prerrealización ( $\left.\mathrm{HL}^{*}\right)$ (Toledo, 2000). No obstante, los resultados contradicen el postulado del modelo métrico autosegmental en relación a la sincronización de la sílaba tónica con el pico tonal (Sosa, 1999; Face, 2002; entre otros).

En la duración, solamente las tónicas presentan DMP, dato que está en línea con lo reportado por Fernández Planas y Martínez Celdrán (2003) quienes también basan sus resultados en las DMP observadas entre vocales pretónicas-tónicas y tónicas-postónicas. Sin embargo, en la duración hay un porcentaje de casos mayor en las declarativas que en las interrogativas. En la intensidad, las declarativas presentan un porcentaje mayor de DMP que las interrogativas en las tónicas al compararlas con las postónicas. En resumen, la duración se muestra como una propiedad de la producción de los sonidos que está específicamente relacionada con las sílabas tónicas independientemente de la modalidad oracional o la estructura sintagmática. Sin embargo, como se verá enseguida, los parámetros se asocian dependiendo de la intención lingüística del hablante, esto es, si desea producir un 
enunciado declarativo o interrogativo.

Respecto a la primera pregunta de investigación que se centra en la existencia o no de asociaciones entre los tres parámetros prosódicos, los resultados confirman la asociación entre éstos y, además, muestran asociaciones en algunas ocasiones en la misma vocal, con un porcentaje mayor en las tónicas que en las pretónicas y postónicas, y en otras las asociaciones se presentan entre vocales adyacentes. Por tanto, para identificar la conformación prosódica de un elemento vocálico necesariamente los valores máximos de los tres parámetros no deben recaer en una misma vocal, sino que hay un patrón de asociación entre los diferentes parámetros acústicos que le permiten al oyente extraer la prosodia del enunciado. En un trabajo llevado a cabo por Ladd, Mennen y Schepman (2000), los autores mostraron que el alineamiento del valor máximo de la $\mathrm{F} 0$ en holandés se veía afectado por la longitud fonológica de la sílaba acentuada. A pesar de la duración real de las vocales, el valor máximo del acento se presentaba antes en sílabas que contenían vocales fonológicamente más largas que en sílabas que contenían vocales fonológicamente más cortas. Desde esta perspectiva, los autores argumentaron que la variabilidad del alineamiento del valor máximo en holandés podía ser parcialmente explicada en términos de anclar este valor al lado derecho de la sílaba, es decir, en el lado derecho de la vocal acentuada, cuando la sílaba contiene vocales fonológicamente largas, o en la consonante siguiente cuando la sílaba contiene vocales fonológicamente cortas. Otro ejemplo se puede encontrar en el estudio realizado en italiano por D'Imperio (2000), quien encontró resultados similares a pesar de que en esta lengua, al igual que en el español, la duración no presenta un rasgo fonológico. Asimismo, Escandel-Vidal (2011) mostró que el incremento en la duración no necesariamente implica una mayor F0, sino que ésta puede correlacionar con un tono bajo. La inferencia que subyace a estos resultados es que no es necesario que los valores máximos de todos los patrones acústicos recaigan en una sola sílaba, pero sí que se presente un patrón de asociación, como se ha evidenciado en los resultados descritos en el presente trabajo. Los fenómenos prosódicos, según Silverman (1990), son el resultado de la señal acústica como de la estructura mental. Por tal motivo, desde el punto de vista de la recepción, el mensaje no se ve afectado porque, a pesar de las características acústicas emitidas, el receptor está preparado cognitivamente para descodificar determinados patrones.

Ahora bien, como se puede observar en la segunda parte de los resultados, en la contingencia entre F0 y duración se evidenciaron asociaciones significativas entre Pr-T y también entre T-Ps, aunque como ya ha quedado claro la duración de la tónica siempre presentó DMP con sus adyacentes. Por tal motivo, al menos en el presente estudio, no se puede sugerir que el desplazamiento de la F0 se deba al tiempo que necesita para lograr su valor máximo (Ladd et al, 2000; Prieto, Van Santen y Hirschberg, 1995), pero sí se sugiere que este patrón se presenta porque 
subyace a la propia lengua. No obstante, estos resultados se presentan con cautela debido a que hacen falta más estudios para confirmarlos.

La segunda pregunta de investigación consiste en observar si estas asociaciones establecen diferencias entre las oraciones declarativas y las interrogativas en función de los sintagmas. Los resultados en conjunto muestran que las asociaciones que se dan en cada contingencia sí diferencian ambas modalidades.

En primer lugar, en el SN en relación a las DMP entre Pr-T, hay que destacar que en los tres tipos de contingencias se presenta una asociación significativa entre los diferentes parámetros en ambas modalidades oracionales. Los resultados muestran que ambas modalidades presentan el mismo patrón de asociación en las contingencias entre la F0-intensidad (coocurren cuando la F0 es mayor en la Pr y la intensidad es mayor en la T) y duración e intensidad (ambas coocurren en las T), pero no en F0-duración. La diferencia consiste en que en la modalidad declarativa las asociaciones entre la F0 y duración se presentan en la tónica, mientras que en la modalidad interrogativa ambos parámetros coocurren cuando la F0 de la pretónica es mayor que la tónica y, a su vez, la tónica presenta una duración perceptivamente mayor que la pretónica. Desde esta perspectiva, la diferencia entre ambas modalidades se pueden evidenciar en la contingencia entre la F0 y la duración.

En el SN en relación a las DMP entre T-Ps en las contingencias F0-duración ambas modalidades presentan un patrón similar, esto es, ambos parámetros coocurren cuando la mayor duración recae en la tónica y la mayor frecuencia en la postónica. En la contingencia F0-intensidad los parámetros coocurren en la postónica de la modalidad interrogativa, en la modalidad declarativa no hay ningún tipo de asociación significativa. Por tanto, la diferencia entre ambas modalidades se puede ubicar en la contingencia entre F0 e intensidad.

En el SP en relación a las DMP entre Pr-T en las contingencias F0-duración se encuentran asociaciones en la tónica de la modalidad interrogativa, en la modalidad declarativa no se encuentra ningún tipo de asociación significativa. En las contingencias F0-intensidad ambas modalidades presentan un patrón similar, esto es, ambos parámetros coocurren en la tónica. Así, las diferencias entre ambas modalidades se pueden ubicar en las contingencias entre F0 y duración.

En el SP en relación a las DMP entre T-Ps en las contingencias F0-intensidad se encuentran asociaciones en la tónica de la modalidad interrogativa, en la modalidad declarativa no se halla ningún tipo de asociación significativa. Por tanto, la diferencia entre ambas modalidades se ubica en la contingencia entre F0 e intensidad.

En términos generales, se concluye que existe un patrón de asociación entre los tres parámetros prosódicos mediatizado por la intención del hablante, esto es, si es declarativa o interrogativa. Además, este estudio ha mostrado la relevancia de usar las DMP puesto que permite identificar aquellas diferencias que son 
percibidas como significativas por el sistema cognitivo. No obstante, en el futuro sería interesante construir un modelo que permitiera observar la influencia de un parámetro en otro.

\section{REFERENCIAS}

Bolinger, Dwight y Hodapp, Marion. (1961). Acento melódico, acento de intensidad. BFUCh, 13, 33-48.

Cabrera, Franchon. (1995). Stress and Intonation in Spanish for affirmative and interrogative sentence. Proceedings of Eurospeech, 95, 2085-2088.

Candia, Luis; Urrutia, Hernán y Fernández, Teresa. (2006). Rasgos acústicos de la prosodia acentual del español. Boletín de Filología, Tomo XLI, 11-14.

Canellada, María Josefa y Madsen, Jhon Kuhlmann. (1987). Pronunciación del español. Lengua hablada y literaria. Madrid: Castalia.

Contreras, Heles. (1963). Sobre el acento en español. Boletín de Filología, XV, 223-237.

Cuervo, Rufino José. (1954). Notas a la Gramática de Bello. Obras, 1, Bogotá.

D'Imperio, Mariapaola. (2000). The role of perception in defining tonal targets and their alignment. Tesis doctoral. Ohio, Estados Unidos: Universidad de Ohio.

Dorta, Josefa; Hernández, Beatriz y Díaz, Chaxiraxi. (2009). Interrogativas absolutas: relación entre F0, duración e intensidad. Estudios de Fonética Experimental 18, 123-144.

Dorta, Josefa; Díaz, Chaxiraxi; Hernández, Beatriz; Jorge, Carolina; y Martín Gómez, Juan. (2013). El marco de la investigación: aspectos metodológicos. En: Dorta, Josefa (Ed.) Estudio comparativo preliminar de la entonación de Canarias, Cuba y Venezuela, (53-82). Santa Cruz de Tenerife: La Página Ediciones.

Escandell-Vidal, Victoria. (2011). Verum focus y prosodia: cuando la duración (sí que) importa. Oralia, 14, 181-201.

Face, Timothy. (2002): Spanish evidence for pitch-accent structure. Linguistics, 40, 319-345.

Fernández Planas, Ana María y Martínez Celdrán, Eugenio. (2003). El tono fundamental y la duración: Dos aspectos de la taxonomía prosódica en dos modalidades de habla (enunciativa e interrogativa) del español. Estudios de Fonética Experimental, 12, 165-200.

Garrido, Juan; Llisterri, Joaquim; De la Mota, Carme y Ríos, Antonio. (1995). Estudio comparado de las características prosódicas de la oración simple en español en dos modalidades de lectura. Elejabetia, A.; Iribar, A., (Eds.) Phonetica. Trabajos de fonética experimental, (173-194). Bilbao: Universidad de Deusto (Serie Lingüística, 6). 
Haberman, Shelby. (1973). The analysis of residuals in cross-classification tables. Biometrics, 29, 205-20.

Ladd, Robert; Mennen, Ineke y Schepman, Astrid. (2000). Phonological conditioning of peak alignment in rising pitch accents in Dutch. Journal of the Acoustical Society of America, 107, 2685-2696.

Llisterri, Joaquim; Machuca, María; De la Mota, Carme; Riera, Monserrat y Ríos, Antonio. (2005). La percepción del acento léxico en español. Filología y lingüistica. Estudios ofrecidos a Antonio Quilis. (pp. 271-97). Madrid: Consejo Superior de Investigaciones Científicas - Universidad Nacional de Educación a Distancia - Universidad de Valladolid.

López, María Jesús; Muñiz, Carmen; Díaz, Liliana; Corral, Norberto; Brezmes, David y Alvarellos, Mercedes. (2007). Análisis y representación de la entonación. Replanteamiento metodológico en el marco del proyecto AMPER. En Dorta, Josefa (Ed.): La prosodia en el ámbito lingüístico románico (17-34). Santa Cruz de Tenerife, España: La Página Ediciones.

Marrero, Victoria. (2008). La fonética perceptiva. Trascendencia lingüística de mecanismos neuropsicofisiológicos. Estudios de Fonética Experimental 17, $207-$ 245.

Martín, José Antonio. (2010). Estudio acústico de las variantes de ch en Tenerife en comparación con la alveolopalatal castellana. Estudios de Fonética Experimental, 19, 165-203.

Martínez Celdrán, Eugenio y Fernández Planas, Ana María. (2006). Hacía una geoprosodia de las lenguas íbero-romances en la "Web". Letras de Hoje, 41, $9-22$.

Martínez Celdrán, Eugenio y Fernández Planas, Ana María. (2013). Manual de Fonética Española. Barcelona: Ariel.

Méndez Seijas, Jorge. (2010). Interacción de los parámetros acústicos, duración y frecuencia fundamental en frases declarativas neutras e interrogativas absolutas de los andes venezolanos. Estudios de Fonética Experimental, 19, 147-164.

Mora Gallardo, Elsa (1998). Acústica del acento español en su variedad venezolana. Lengua y Habla, 3, 70-78.

Muñetón, Mercedes. (2016). La F0, duración e intensidad de las oraciones interrogativas absolutas en un informante varón de Medellín. Estudios de fonética Experimental, XXV, 167-192.

Muñetón, Mercedes y Dorta, Josefa. (2015). La entonación declarativa e interrogativa en el español colombiano de Medellín: voz femenina vs. masculina. Boletín de Filología, 2, 103-122.

Navarro Tomás, Tomás. (1963). Manual de pronunciación española (11 edición). Madrid: CSIC.

Nooteboom, Sieb. (1997). The prosody of speech: Melody and rhythm. Hardcastle, William y John Laver (eds.), The Handbook of Phonetic Sciences 
(640-673). Oxford: Blackwell.

Pamies Bertrán, Antonio y Fernández Planas, Ana María. (2006). La percepción de la duración vocálica en español. Luque Durán, J. D. (Ed.), Actas del V Congreso Andaluz de Lingüistica General, Homenaje al Profesor José Andrés de Molina Redondo I (501-512). Granada: Granada Lingvistica-Ediciones Método.

Pamies Bertrán, Antonio; Fernández Planas, Ana María; Martínez Celdrán, Eugenio; Ortega Escandell, Alicia y Amorós Céspedes, Mari Cruz. (2001). Umbrales tonales en español peninsular. Actas del II Congreso de Fonética Experimental (272-278). Sevilla, España: Universidad de Sevilla.

Prieto, Pilar; Van Santen, Jan; y Hirschberg, Julia. (1995). Tonal alignment patterns in Spanish. Journal of Phonetics, 23, 429-451.

Quilis, Antonio (1988). Fonética acuistica de la lengua española. Madrid: Gredos.

Rietveld, Toni y Gussenhoven, Carlos. (1985). On the relation between pitch excursión size and prominence. Journal of Phonetics, 13, 299-308.

Romano, Antonio y Michel Contini. (2001). Un progetto di Atlante geoprosódico multimediale delle varieta linguistiche romanze. En Magno Caldognetto, Pelachaud y Piero Cosi, Multimodalità e Multimedialità nella Comunicazione, Atti delle XI giornate di Studio del gruppo di Fonetica Sperimentale, dell'Ass pp. 121-126. Italiana di Acustica (Padova, 9 nov.-1 dic. 2000), Padova: Unipress.

Silverman, Kim. (1990). The separation of prosodies. John Kigston y Mary Bechian (Eds.) Papers in Laboratory Phonology I (139-151). Cambridge: Cambridge University Press.

Sosa, Juan Manuel. (1999): La entonación del español. Su estructura fónica, variabilidad y dialectología. Madrid: Cátedra.

Toledo, Guillermo. (2000). Taxonomía tonal del español. Language Design, 3, $1-20$.

Urrutia, Hernán. (2007). La naturaleza del acento en español: nuevos datos y perspectivas. Revista de Lingüistica Teórica y Aplicada, 45, 135-142.

Valiente, Ana Belén. (2012). Aplicación de la teoría de la optimidad al consonantismo del habla del consejo de Casares de las Hurdes. Anuario de estudios filológicos, 35, 235-253. 